\title{
DIFFERENCES IN THE PREVALENCE AND CHARACTERISTICS OF DISTRACTED DRIVING IN EIGHT
} COUNTRIES, 2011

doi:10.1136/injuryprev-2012-040580g.5

R Naumann*, A Dellinger. National Center for Injury Prevention and Control, Centers for Disease Control and Prevention, USA

Background Distraction is a risk factor for traffic crashes that is becoming an increasing global concern; however, its prevalence is not well documented.

Aims/Objectives/Purpose To examine the prevalence and characteristics of those who talk or text using cellular devices while driving in Belgium, France, Germany, the Netherlands, Portugal, Spain, the UK, and the USA.

Methods Online survey of adults aged 18-64 in the USA ( $N=3294)$ and seven European countries ( $N=8955)$, weighted to the age and sex distributions of each country. Respondents reported how often they talk on their cellular phone while driving and how often they read or send text messages or email while driving. We examined differences in prevalence by country, age group, and sex.

Results/Outcomes More than one in four $(27.3 \%$, 95\% CI $25.1 \%$ to $29.4 \%$ ) US adult drivers aged 18-64 reported they 'regularly or fairly 
often' talk on their cellular phone while driving. In Europe, percentages ranged from $20.4 \%$ in Portugal (95\% CI $16.5 \%$ to $24.3 \%$ ) to $7.8 \%$ in the UK (5.9\% to $9.7 \%$ ). Additionally, $7.7 \%$ (6.3\% to $9.0 \%)$ of US drivers aged 18-64 reported that they 'regularly or fairly often' read or send text messages or email while driving. In Europe, percentages ranged from $8.1 \%$ (95\% CI 6.6\% to $9.6 \%$ ) in Belgium to $2.5 \%$ (95\% CI $1.3 \%$ to $3.7 \%$ ) in the UK. There were few differences by sex, but there were differences by age group within most countries.

Significance/Contribution to the Field This study provides recent prevalence estimates of distracted driving-related behaviours in eight countries, which can be used to target high-risk groups for prevention efforts. 